\title{
Finite-pulse waves for efficient suppression of evolving mesoscale dendrites in rechargeable batteries
}

\author{
Asghar Aryanfar $\odot,{ }^{1,2,{ }^{*}}$ Michael R. Hoffmann, ${ }^{1}$ and William A. Goddard III $\odot^{1}$ \\ ${ }^{1}$ California Institute of Technology, 1200 East California Boulevard, Pasadena, California 91125, USA \\ ${ }^{2}$ Bahçeşehir University, 4 Çıră̆an Cad, Beşiktaş, Istanbul, Turkey 34353
}

(Received 16 July 2019; published 9 October 2019)

\begin{abstract}
The ramified and stochastic evolution of dendritic microstructures has been a major issue on the safety and longevity of rechargeable batteries, particularly for the utilization of high-energy metallic electrodes. We analytically develop criteria for the pulse characteristics leading to the effective halting of the ramified electrodeposits grown during extensive timescales beyond inter-ionic collisions. Our framework is based on the competitive interplay between diffusion and electromigration and tracks the gradient of ionic concentration throughout the entire cycle of pulse-rest as a critical measure for heterogeneous evolution. In particular, the framework incorporates the Brownian motion of the ions and investigates the role of the geometry of the electrodeposition interface. Our experimental observations verify the analytical developments, where the dimension-free developments allows the application to the electrochemical systems of various scales.
\end{abstract}

DOI: 10.1103/PhysRevE.100.042801

\section{INTRODUCTION}

Metallic anodes such as lithium, sodium, and zinc are arguably highly attractive candidates for use in high-energy and high-power density rechargeable batteries [1-3]. In particular, lithium metal possesses the lowest density and smallest ionic radius which provides a very high gravimetric energy density and possesses the highest electropositivity $\left(E^{0}=-3.04 \mathrm{~V}\right.$ versus standard hydrogen electrode) that likely provides the highest possible voltage, making it suitable for high-power applications such as electric vehicles $\left(\rho=0.53 \mathrm{~g} \mathrm{~cm}^{-3}\right)[4,5]$. During the charging, the fast-pace formation of microstructures with relatively low surface energy from Brownian dynamics leads to the branched evolution with high surface to volume ratio [6]. The quickening treelike morphologies could occupy a large volume, possibly reach the counterelectrode, and short the cell. Additionally, they can also dissolve from their thinner necks during subsequent discharge period. Such a formation-dissolution cycle is particularly prominent for the metal electrodes due to lack of intercalation ${ }^{1}$ [7]. Previous studies have investigated various factors on dendritic formation such as current density [8], electrode surface roughness [9-11], impurities [12,13], solvent and electrolyte chemical composition $[14,15]$, electrolyte concentration [16], utilization of powder electrodes [17] and adhesive polymers [18], temperature [19], guiding scaffolds [20,21], capillary pressure [22], cathode morphology [23], and mechanics [24,25]. Some of conventional characterization techniques used include NMR [26] and MRI [27]. Recent studies also have shown the necessity of stability of solid electrolyte interphase (SEI) layer for controlling the nucleation and growth of the branched medium [28,29].

\footnotetext{
*aryanfar@caltech.edu.

${ }^{1}$ Intercalation: diffusion into inner layer as the housing for the charge, as opposed to depositing in the surface.
}

An earlier model of dendrites had focused on the electric field and space charge as the main responsible mechanism [30], while the later models focused on ionic concentration causing the diffusion limited aggregation (DLA) [31-33]. Both mechanisms are part of the electrochemical potential $[34,35]$, indicating that each could be dominant depending on the localizations of the electric potential or ionic concentration within the medium. Nevertheless, their interplay has been explored rarely, especially in continuum scale and realistic time intervals, matching scales of the experimental time and space.

Dendrites instigation is rooted in the nonuniformity of electrode surface morphology at the atomic scale combined with Brownian ionic motion during electrodeposition. Any asperity in the surface provides a sharp electric field that attracts the upcoming ions as a deposition sink. Indeed, the closeness of a convex surface to the counter electrode, as the source of ionic release, is another contributing factor. In fact, the same mechanism is responsible for the further semi-exponential growth of dendrites in any scale. During each pulse period the ions accumulate at the dendrites tips (unfavorable) due to high electric field in convex geometry and during each subsequent rest period the ions tend to diffuse away to other less concentrated regions (favorable). The relaxation of ionic concentration during the idle period provides a useful mechanism to achieve uniform deposition and growth during the subsequent pulse interval. Such dynamics typically occurs within the double layer (or stern layer [36]) which is relatively small and comparable to the Debye length. In high charge rates, the ionic concentration is depleted and concentration on the depletion reaches zero [37]; Nonetheless, our continuum-level study extends to larger scale, beyond the double layer region [38].

Pulse method has been qualitatively proved as a powerful approach for the prevention of dendrites [39], which has previously been utilized for uniform electroplating [40]. In the preceding publication we have experimentally found that the 
optimum rest period correlates well with the relaxation time of the double layer for the blocking electrodes [41] which is interpreted as the RC time of the electrochemical system [42]. We have explained qualitatively how relatively longer pulse periods with identical duty cycles $D$ (or idle ratio $\gamma$ ) will lead to longer and more quickening growing dendrites. We developed coarse grained computationally affordable algorithm that allowed us reach to the experimental timescale (ms). Additionally, in the recent theoretical work we indicated that there is an analytical criterion for the optimal inhibition of growing dendrites [43].

In this paper, we elaborate further in the range of acceptable duty cycle $D$ for suppression of stochastically grown dendrites and we develop an insight for the effective rest period on the curved boundary. Subsequently we carry out experimental investigation to verify our analytical developments on the pulse parameters. We perform dimensional analysis to set our formulation applicable to the large range of electrochemical devices.

\section{METHODOLOGY}

The electrochemical flux is generated either from the gradients of concentration $(\vec{\nabla} C)$ or electric potential $(\vec{\nabla} \phi)$. In the ionic scale, the regions of higher concentration tend to collide and repel more and, given enough time, diffuse to lower concentration zones, following Brownian motion. In the continuum scale, such intercollisions could be added up and represented by the diffusion length $\delta \overrightarrow{\mathbf{r}}_{D}$ as [41] ${ }^{2}$

$$
\delta \overrightarrow{\mathbf{r}}_{D}=\sqrt{2 D^{+} \delta t} \hat{\mathbf{g}},
$$

where $\overrightarrow{\mathbf{r}}_{D}$ is diffusion displacement of individual ion, $D^{+}$ is the ionic diffusion coefficient in the electrolyte, $\delta t$ is the coarse time interval, ${ }^{3}$ and $\hat{\mathbf{g}}$ is a normalized vector in random direction, representing the Brownian dynamics. The diffusion length represents the average progress of a diffusive wave in a given time, obtained directly from the diffusion equation [45].

However, ions tend to acquire drift velocity in the electrolyte medium when exposed to electric field and during the given time $\delta t$ their progress $\delta \overrightarrow{\mathbf{r}}_{M}$ is given as

$$
\delta \overrightarrow{\mathbf{r}}_{M}=\mu^{+} \overrightarrow{\mathbf{E}} \delta t,
$$

where $\mu^{+}$is the mobility of cations in electrolyte, and $\overrightarrow{\mathbf{E}}$ is the local electric field, which is the gradient of electric potential $(\overrightarrow{\mathbf{E}}=-\vec{\nabla} \phi)$. Therefore, the total effective displacement $\delta \overrightarrow{\mathbf{r}}$ with neglecting convection ${ }^{4}$ would be

$$
\delta \overrightarrow{\mathbf{r}}=\delta \overrightarrow{\mathbf{r}}_{D}+\delta \overrightarrow{\mathbf{r}}_{M},
$$

as represented in the Fig. 1. The pulse charging in its simplest form consists of trains of square active period $t_{\mathrm{ON}}$, followed

\footnotetext{
${ }^{2}$ The diffusion coefficient $D^{+}$is generally concentration dependent [44], due to electroneutrality within the considerable space in the domain and we assume it is constant in the range considered.

${ }^{3} \delta t=\sum_{i=1}^{n} \delta t_{i}$, where $\delta t_{k}$ is the intercollision time, typically in the range of $f s$.

${ }^{4}$ Since Rayleigh number $\mathrm{Ra}$ is highly dependent to the thickness (i.e., $\mathrm{Ra} \propto l^{3}$ ), for a thin layer of electrodeposition we have $\mathrm{Ra}<1500$, and thus the convection is negligible [46].
}

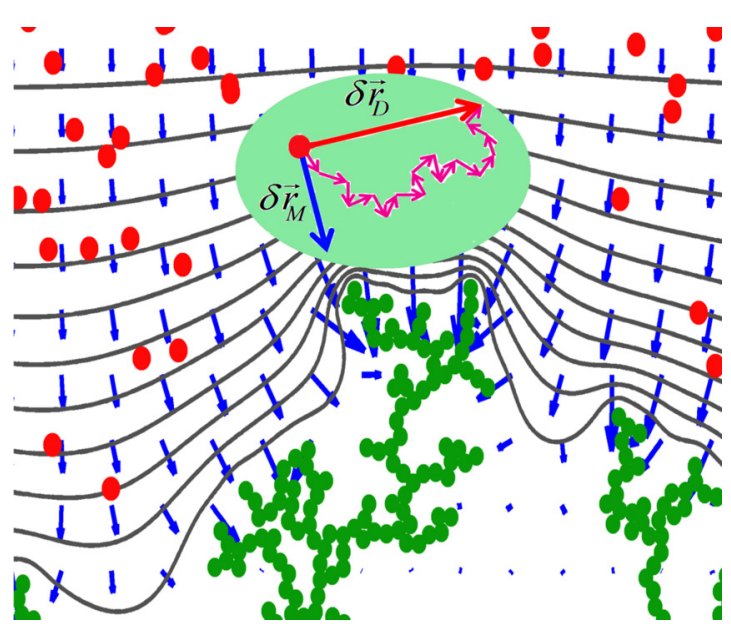

FIG. 1. The transport elements in the coarse scale of time and space.

by a square rest interval $t_{\mathrm{OFF}}$ in terms of current $I$ or voltage $V$ as shown in Fig. 2. The period $P=t_{\mathrm{ON}}+t_{\mathrm{OFF}}$ is the time lapse of a full cycle. Hence, the pulse frequency $f$ is

$$
f=\frac{1}{t_{\mathrm{ON}}+t_{\mathrm{OFF}}},
$$

and the duty cycle $\boldsymbol{D}$ represents the fraction of time in the period $P$ that the pulse is active:

$$
\mathbf{D}=f t_{\mathrm{ON}} .
$$

Based on the Eqs. (4) and (5), defining two parameters will uniquely characterize the pulse charge. We choose them as duty cycle $D$ (Sec. II A) and the relaxation (i.e., rest) period $t_{\mathrm{OFF}}(\mathrm{Sec}$. II B) as follows next.

\section{A. Optimum duty cycle}

The vector sum in Eq. (3) indicates that the diffusion, as a random walk, can either contribute to electromigration or prevents its progress, depending on the local orientation of the gradients of concentration and electric potential $\{\vec{\nabla} C, \vec{\nabla} \phi\}$. From Fig. 1 it is visually obvious that the sum of individual diffusional displacements after the $n$ number of collisions within the time interval $\delta t$ always is larger (or equal to) than the on-step displacement of diffusion front during the entire

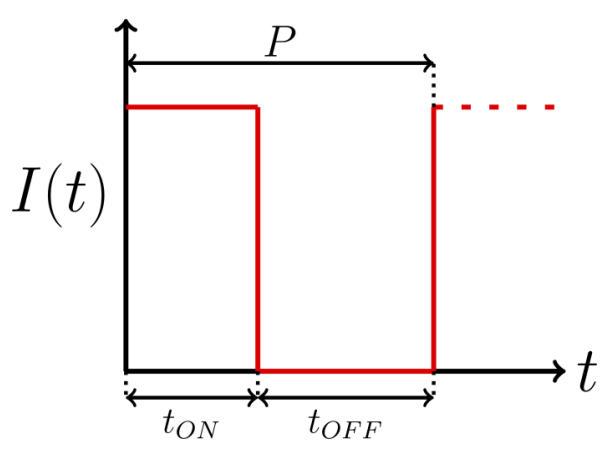

FIG. 2. Square pulse wave. 
coarse time interval $\delta t=\sum_{i=1}^{n} \delta t_{i}$ as

$$
\sum_{i=1}^{n} \sqrt{2 D^{+} \delta t_{i}} \geqslant \sqrt{2 D^{+} \sum_{i=1}^{n} \delta t_{i}}
$$

We verify Eq. (6) by induction. The equation is obvious for value of $n:=1$, therefore we need to prove that if Eq. (6) is true for the $n:=k$, then it should be true for $n:=k+1$ :

$$
\sum_{i=1}^{k+1} \sqrt{2 D^{+} \delta t_{i}} \geqslant \sqrt{2 D^{+} \sum_{i=1}^{k+1} \delta t_{i}}
$$

Assuming that $\delta t_{i}=\delta t$ (i.e., equal segmentation) the inequality Eq. (7) can be broken down as

$$
\sum_{i=1}^{k} \sqrt{2 D^{+} \delta t_{i}}+\sqrt{2 D^{+} \delta t} \geqslant \sqrt{2 D^{+} \sum_{i=1}^{k} \delta t_{i}+2 D^{+} \delta t .}
$$

Taking to the power 2, with simplification, we get the following:

$$
2 k(k+1) D^{+} \delta t \geqslant 0 \checkmark,
$$

which means that Eq. (7) is true for any consecutive value of $k \rightarrow k+1$ and therefore indefinitely for any $k \in \mathbb{N}$. In fact, Eq. (6) represents the extended version of triangle inequality in terms of mean-square diffusion distance [47]. During each pulse period $t_{\mathrm{ON}}$, both diffusion and migration are active for the ionic displacements. Therefore, depending on their individual orientation they can help or hurt each other. Thus, the range of ionic displacement $|\delta \overrightarrow{\mathbf{r}}|_{\text {ON }}$ in the pulse period is obtained as

$$
\mu^{+} \overrightarrow{\mathbf{E}} \delta t-\sum_{i=1}^{n} \sqrt{2 D^{+} \delta t_{i}} \leqslant|\delta \overrightarrow{\mathbf{r}}|_{\mathrm{ON}} \leqslant \mu^{+} \overrightarrow{\mathbf{E}} \delta t+\sum_{i=1}^{n} \sqrt{2 D^{+} \delta t_{i}},
$$

where $\mu^{+}$and $D^{+}$are the mobility and the diffusion coefficient of local ions and $\overrightarrow{\mathbf{E}}$ is the local electric field, respectively. For Eq. (9) to be valid, considering Eq. (6), one must have

$$
\mu^{+} \overrightarrow{\mathbf{E}} \delta t-\sqrt{2 D^{+} \delta t} \leqslant|\delta \overrightarrow{\mathbf{r}}|_{\mathrm{ON}} \leqslant \mu^{+} \overrightarrow{\mathbf{E}} \delta t+\sqrt{2 D^{+} \delta t} .
$$

Such a random walk is succeeded with the idle period $t_{\mathrm{OFF}}$ where the diffusion is the sole drive for the relaxation. To have uniform electrodeposition, the average progress of diffusive wave in the rest period $t_{\mathrm{OFF}}$ has to be competitive enough with the pulse interval $t_{\mathrm{ON}}$, hence

$$
\sqrt{2 D^{+} t_{\mathrm{OFF}}} \geqslant \mu^{+} \overrightarrow{\mathbf{E}} t_{\mathrm{ON}} \pm \sqrt{2 D^{+} t_{\mathrm{ON}}} .
$$

Without further look into Eq. (11), it is obvious that $t_{\mathrm{OFF}} \geqslant$ $t_{\mathrm{ON}}$. For simplification, we define the idle ratio as $\gamma:=\frac{t_{\mathrm{OFF}}}{t_{\mathrm{ON}}}$ and further elaboration leads to

$$
\gamma \pm 2 \sqrt{\gamma}+1-\frac{\mu^{+}|\overrightarrow{\mathbf{E}}|^{2}}{2 R T} t_{\mathrm{ON}} \geqslant 0
$$

The solution to Eq. (12) represents the idle ratio for effective fading as

$$
\gamma \geqslant\left(1 \pm|\overrightarrow{\mathbf{E}}| \sqrt{\frac{2 \mu^{+} t_{\mathrm{ON}}}{R T}}\right)^{2}
$$

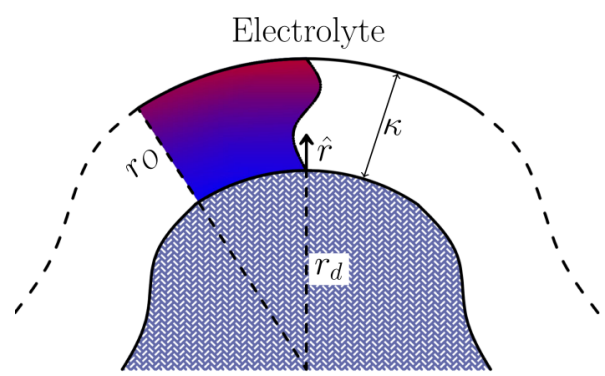

Dendrite

FIG. 3. The curved dendrites with the concentration gradient the vicinity of surface.

and the duty cycle $D$ in terms of the idle ratio $\gamma$ is obtained as

$$
\mathbf{D}=\frac{t_{\mathrm{ON}}}{t_{\mathrm{ON}}+t_{\mathrm{OFF}}}=\frac{1}{1+\gamma} \text {. }
$$

Noting the Einstein relationship $\left(D^{+}=\mu^{+} R T\right)$, where $R$ is the universal gas constant and $T$ is the temperature. the range of acceptable duty cycle $D$ would be

$$
\mathbf{D} \leqslant \frac{1}{\left(1+\frac{|\overrightarrow{\mathbf{E}}|}{R T} \sqrt{\frac{D^{+}}{2 f}}\right)^{2} \pm 1} .
$$

\section{B. Optimum relaxation}

The dendritic tip in fact attracts a significant number of ions due to high electric field. Given sufficient time, such ionic concentration profile can disappear in the vicinity of curved electrodeposits during subsequent idle period. Therefore, the relaxation of concentration plays a key role for preventing dendritic deposition. In fact, the oscillation of the concentration gradient repeatedly occurs during each pulse-rest period [37]. Herein, we address a time measure for concentration relaxation in the continuum scale with the curved boundary rising from the tip of growing dendrites.

The schematics of the convex dendrites is shown in Fig. 3 with the surrounding double layer of thickness of $\kappa$ and the outer electro-neutral medium. The color gradient represents the concentration profile in the double-layer region. The radius of curvature $r_{d}$ could vary from atomic radius $\left(r_{d} \approx\right.$ $\left.r_{\mathrm{Li}^{+}} \rightarrow 10^{-9} \mathrm{~m}\right)$ [41] to nearly flat surfaces $\left(r_{d} \rightarrow \infty\right)$. Such a wide range makes orders-of-magnitude of difference in the electric field and concentration dynamics, making it critical factor to consider. We define the normalized dendrite radial distance $\hat{r} \in[0,1]$ from the tip as

$$
\hat{r}:=\frac{r-r_{d}}{\kappa},
$$

where $r$ is the center of curvature. Subsequently, we can define the normalized concentration $\hat{C} \in[0,1]$ as

$$
\hat{C}(\hat{r}):=\frac{C(\hat{r})}{C_{\infty}},
$$

where the index $\infty$ represent the ambient electroneutral medium. The typical diffusion equation in polar 2D 
coordinates is defined as ${ }^{5}$

$$
\begin{aligned}
\frac{\partial C}{\partial t} & =\vec{\nabla} \cdot\left(D^{+} \vec{\nabla} C\right) \\
& =\left(\frac{\partial}{\partial r}+\frac{1}{r} \frac{\partial}{\partial \theta}\right)\left[D\left(\frac{\partial C}{\partial r}+\frac{1}{r} \frac{\partial C}{\partial \theta}\right)\right] \\
& =D^{+}\left(\frac{\partial^{2} C}{\partial r^{2}}+\frac{1}{r} \frac{\partial C}{\partial r}\right),
\end{aligned}
$$

where $r$ and $\theta$ are the radial and azimuthal directions. Using the chain derivative and noting Eq. (17), we get

$$
\frac{\partial C}{\partial t}=\frac{\partial C}{\partial \hat{C}} \frac{\partial \hat{C}}{\partial t}=C_{\infty} \frac{\partial \hat{C}}{\partial t} .
$$

Respectively, for the radial space derivative is obtained, considering Eq. (16), as

$$
\frac{\partial C}{\partial r}=\frac{\partial C}{\partial \hat{C}} \frac{\partial \hat{C}}{\partial \hat{r}} \frac{\partial \hat{r}}{\partial r}=\frac{C_{\infty}}{\kappa} \frac{\partial \hat{C}}{\partial \hat{r}} .
$$

The second radial derivative is, respectively, obtained as

$$
\begin{aligned}
\frac{\partial^{2} C}{\partial r^{2}} & =\frac{\partial}{\partial r}\left(\frac{\partial C}{\partial r}\right)=\frac{\partial}{\partial r}\left(\frac{C_{\infty}}{\kappa} \frac{\partial \hat{C}}{\partial \hat{r}}\right) \\
& =\frac{C_{\infty}}{\kappa} \frac{\partial}{\partial \hat{r}}\left(\frac{\partial \hat{C}}{\partial \hat{r}}\right) \frac{\partial \hat{r}}{\partial r}=\frac{C_{\infty}}{\kappa^{2}} \frac{\partial^{2} \hat{C}}{\partial \hat{r}^{2}} .
\end{aligned}
$$

Therefore, replacing all the obtained terms from Eqs. (19), (20), and (21) into Eqs. (18) and simplification leads to

$$
\kappa^{2} \frac{\partial \hat{C}}{\partial t}=D^{+}\left(\frac{\partial^{2} \hat{C}}{\partial \hat{r}^{2}}+\frac{\kappa}{r_{d}+\kappa \hat{r}} \frac{\partial \hat{C}}{\partial \hat{r}}\right) .
$$

Regarding the boundary conditions, while the concentration is depleted in the double layer, in the outer boundary $(\hat{r} \rightarrow 1)$ it remains as the ambient value $C_{\infty}$ :

$$
\hat{C}(1, t)=1 .
$$

During the charge period, a constant reduction ionic flux $j$ is fed to the dendrite and, respectively, during the idle period the net sum of the ionic flux between the dendrites and electrolyte is zero due to equilibrium. Therefore,

$$
\begin{aligned}
& \frac{\partial \hat{C}}{\partial \hat{r}}(0, t)=\frac{\kappa}{C_{\infty}} \frac{\partial C}{\partial r}=-\frac{\kappa j}{C_{\infty} D^{+}} \quad \text { Pulse, } \\
& \frac{\partial \hat{C}}{\partial \hat{r}}(0, t)=0 \quad \text { Rest. }
\end{aligned}
$$

Equation (22) can be solved numerically using a finite difference method where the $\hat{C}_{i}^{j}$ represents the concentration in the radial direction $\hat{r}(i)$ and at the time $t(j)$. Performing segmentation in the time $\delta t$ and space $\delta \hat{r}$ and utilizing the scheme of forward-move in time and space (FTFS), we arrive

\footnotetext{
${ }^{5}$ The convection in the azimuthal direction $\hat{\theta}$ is neglected due to below-threshold Rayleigh number $(\mathrm{Ra}<1500)$.
}

at the following:

$$
\begin{aligned}
& \frac{\hat{C}_{i}^{j+1}-\hat{C}_{i}^{j}}{\delta t} \\
& \quad=D^{+}\left[\frac{1}{\kappa^{2}} \frac{\hat{C}_{i+1}^{j}-2 \hat{C}_{i}^{j}+\hat{C}_{i-1}^{j}}{\delta \hat{r}^{2}}+\frac{1}{\kappa\left(r_{d}+\kappa \hat{r}\right)}\left(\frac{\hat{C}_{i+1}^{j}-\hat{C}_{i}^{j}}{\delta \hat{r}}\right)\right] .
\end{aligned}
$$

Equation (25) can be rearranged in terms of individual concentration terms as

$$
\begin{aligned}
C_{i}^{j+1}= & \left(1-\frac{2}{\kappa^{2}} \frac{D^{+} \delta t}{\delta \hat{r}^{2}}-\frac{D^{+} \delta t}{\left(r_{d}+\kappa \hat{r}\right) \kappa \delta \hat{r}}\right) C_{i}^{j} \\
& +\left(\frac{D^{+} \delta t}{\kappa^{2} \delta \hat{r}^{2}}+\frac{D^{+} \delta t}{\left(r_{d}+\kappa \hat{r}\right) \kappa \delta \hat{r}}\right) \hat{C}_{i+1}^{j}+\frac{1}{\kappa^{2}} \frac{D^{+} \delta t}{\delta \hat{r}^{2}} \hat{C}_{i-1}^{j},
\end{aligned}
$$

which can be simplified to the following:

$$
\begin{aligned}
\hat{C}_{i}^{j+1}= & \left(1-\frac{2 Q_{1}}{\delta \hat{r}^{2}}-\frac{\hat{r}}{\delta \hat{r}} Q_{2}\right) \hat{C}_{i}^{j}+\left(Q_{1}+\frac{\hat{r}}{\delta \hat{r}} Q_{2}\right) \hat{C}_{i+1}^{j} \\
& +Q_{1} \hat{C}_{i-1}^{j} .
\end{aligned}
$$

The terms $Q_{1}$ and $Q_{2}$ are the dimension-free quotients, as follows:

$$
\begin{aligned}
Q_{1} & =\frac{D^{+} \delta t}{\kappa^{2}} \\
Q_{2} & =\frac{D^{+} \delta t}{\left(r_{d}+\kappa \hat{r}\right) \kappa \hat{r}} .
\end{aligned}
$$

Equation (27) should possess enough resolution in time $\delta t$ to capture the variations in space $\delta \hat{r}$. Therefore, the stability criterion requires for the coefficient of $\hat{C}_{i}^{j}$ to be nonnegative:

$$
\delta \hat{r}^{2}-\frac{D^{+} \delta t}{\left(r_{d}+\kappa \hat{r}\right) \kappa} \delta \hat{r}-\frac{2 D^{+} \delta t}{\kappa^{2}} \geqslant 0,
$$

which is a parabolic equation in terms of $\delta \hat{r}$. Therefore, noting $\hat{r}_{\max }=1$, we have

$$
\delta \hat{r} \geqslant \frac{D^{+} \delta t}{2 \kappa\left(r_{d}+\kappa\right)} \pm \frac{1}{2} \sqrt{\left(\frac{D^{+} \delta t}{\kappa\left(r_{d}+\kappa\right)}\right)^{2}+8 \frac{D^{+} \delta t}{\kappa\left(r_{d}+\kappa\right)}} .
$$

Looking closer at the term $8 \frac{D^{+} \delta t}{\kappa\left(r_{d}+\kappa\right)}$, the nominator in fact represents the square of the progress for the diffusive wave during the time $\delta t$, which, to be captured, must fall inside the double layer, with the scale of $\kappa$. In other words, $D^{+} \delta t \leqslant$ $\kappa^{2}<\kappa\left(r_{d}+\kappa\right) \Rightarrow \frac{D^{+} \delta t}{\kappa\left(r_{d}+\kappa\right)}<1$. Thus, for Eq. (30) to be true, simplification of the right-hand side will lead to

$$
\delta \hat{r} \geqslant \frac{2 D^{+} \delta t}{\left(r_{d}+\kappa\right) \kappa}
$$

noting the outer radius $r_{O}$ we have $r_{d}+\kappa:=r_{O}$ from Fig. 3. Equation (31) in fact inherits the scale-free time measure for concentration dynamics as

$$
\delta \hat{t}=\frac{D^{+} \delta t}{\kappa\left(\kappa+r_{d}\right)},
$$




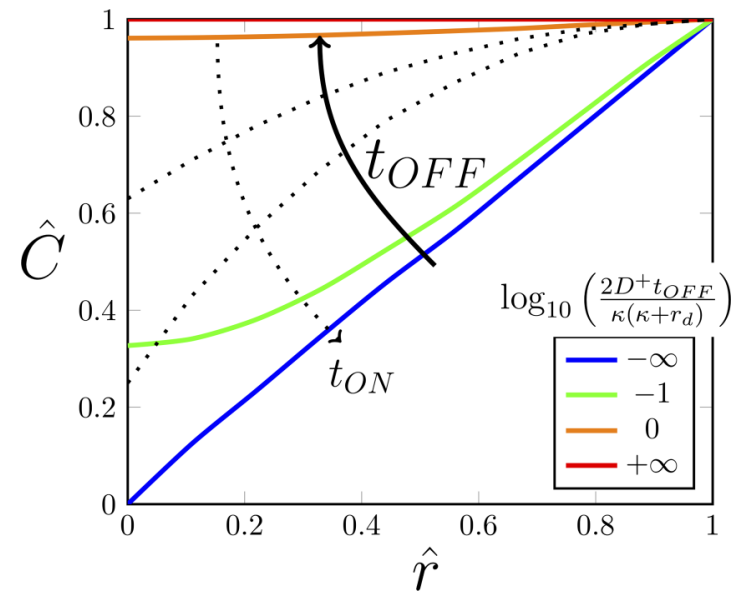

FIG. 4. The concentration profile during the rest period $t_{\mathrm{OFF}}$ (color) and the subsequent pulse interval $t_{\mathrm{ON}}$ (dots).

and the dimension-free space-time criterion for all $\hat{r} \in[0,1]$ is obtained as

$$
\frac{\delta \hat{t}}{\delta \hat{r}}<\frac{1}{2} .
$$

The evolution of concentration profile $\hat{C}$ from Eq. (22) during the entire cycle of pulse-rest has been shown in Fig. 4 with the constants given in Table I.

\section{EXPERIMENTAL}

The dendritic measurements have been carried out in a manually fabricated electrolytic cell [49] that provides the possibility of in situ observation of growing dendrites from the periphery in real time, as shown in Fig. 5(a). The sandwich cell consists of two $\mathrm{Li}^{0}$ foil disk electrodes $(D=1.59 \mathrm{~cm})$ with the interelectrode separation of $L=0.32 \mathrm{~cm}$ by means of a transparent acrylic PMMA housing. The fabricated cells were filled with $0.4 \mathrm{~cm}^{3}$ of $\mathrm{LiPF}_{6}$ in a the stoichiometric compound of $\mathrm{EC}: \mathrm{EMC} \equiv 1: 1$. We performed the operations in an argon-filled glovebox $\left(\mathrm{H}_{2} \mathrm{O}, \mathrm{O}_{2}<0.5 \mathrm{ppm}\right)$.
TABLE I. Simulation parameters.

\begin{tabular}{lcccc}
\hline \hline Parameter & $D^{+}$ & $\kappa$ & $j$ & $r_{d}$ \\
\hline Value & $2.58 \times 10^{-10}$ & 20 & $10^{-4}$ & 20 \\
Unit & $\mathrm{m}^{2} / \mathrm{s}$ & $\mu \mathrm{m}$ & $\mathrm{C} / \mathrm{m}^{2} \mathrm{~s}$ & $\mathrm{~nm}$ \\
Ref. & {$[16]$} & {$[30]$} & {$[48]$} & {$[48]$} \\
\hline \hline
\end{tabular}

Multiple such cells were electrolyzed with current density pulse trains consisting of a range of frequencies $f$, generated by a programmable multichannel charger. After the passage of $48 \mathrm{mAh}\left(\approx 173^{\circ} \mathrm{C}\right)$ through the cells, three images within the periphery of $120^{\circ}$ were taken by means of Leica M205FA optical microscope through the acrylic separator. The image processing algorithm given in Fig. 5(c) is described as follows:

(1) The RGB image is read to the program by three values of $\{R, G, B\} \in[0,255]$ and has been converted to a gray-scale image $I$ with individual values of range $I_{i, j} \in[0,1]$.

(2) The image is binarized from Otsu's method. For this purpose a critical grayness threshold $I_{c}$ has been chosen to approximate the gray-scale image $I$ with a binarized image $J$ as follows:

$$
J_{i, j}=\left\{\begin{array}{ll}
1 & I_{i, j} \geqslant I_{c} \\
0 & I_{i, j}<I_{c}
\end{array} .\right.
$$

The threshold value $I_{c}$ has been chosen to minimize the weighted intraclass variance $\sigma^{2}$ defined as

$$
\begin{aligned}
& \sigma^{2}=\omega_{0} \sigma_{0}^{2}+\omega_{1} \sigma_{1}^{2}, \\
& \omega_{0}+\omega_{1}=1,
\end{aligned}
$$

where $\omega_{0}$ and $\omega_{1}$ are the total fraction of element divided by the value of $I_{c}$ and $\sigma_{0}^{2}$ and $\sigma_{1}^{2}$ are their respective variances [50].

(3) The circular sandwich cell with the radius $R$ has been divided into three arcs with the angle of $\frac{2 \pi}{3}$ and width incremental length of $\delta x$, which is supposed to be projected to a 2D plane with the incremental width of $\delta x^{\prime}$. From Fig. 5(a), due to geometry, we have $x=\frac{D}{2} \sin (\theta), \rightarrow d x=\frac{D}{2} \cos (\theta) d \theta$, where

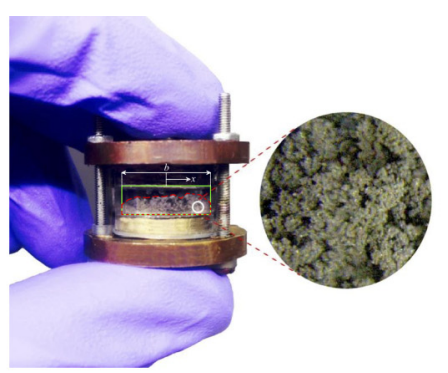

(a)

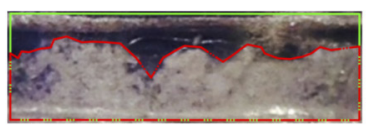

$\mathbf{D}=0.32, \bar{\lambda}=0.81$

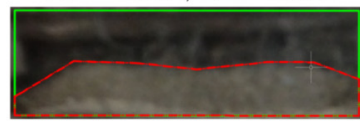

$\mathbf{D}=0.18, \bar{\lambda}=0.45$

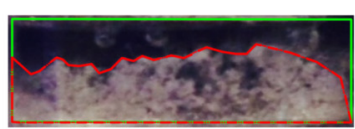

$\mathbf{D}=0.24, \bar{\lambda}=0.68$

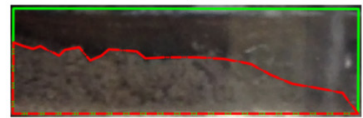

$\mathbf{D}=0.12, \bar{\lambda}=0.41$

(b)

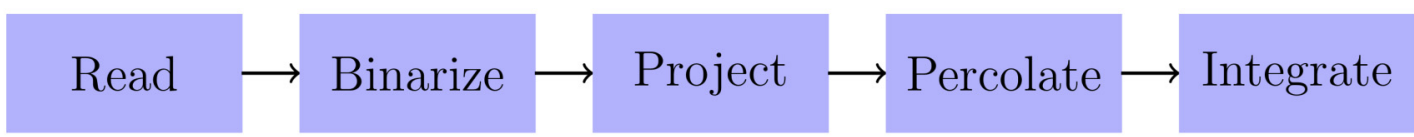

(c)

FIG. 5. Experimental procedure. (a) Naked-eye observation of dendrites [19], (b) Sample tracking of the suppression for $f=100 \mathrm{~Hz}$, and (c) Extracting the dendrite measure from experimental images. 
TABLE II. Experimental parameters.

\begin{tabular}{|c|c|c|c|c|c|c|c|}
\hline Parameter & $f$ & $i$ & $l$ & $R$ & $T$ & $C_{\infty}$ & $|\tilde{\mathbf{E}}|_{\max }{ }^{\mathrm{a}}$ \\
\hline Value unit & $\begin{array}{c}\{25,40,100,250,1000\} \\
\mathrm{mHz}\end{array}$ & $\begin{array}{c}1 \\
\mathrm{~mA} / \mathrm{cm}^{2}\end{array}$ & $\begin{array}{c}3.175 \\
\mathrm{~mm}\end{array}$ & $\begin{array}{c}0.795 \\
\mathrm{~cm}\end{array}$ & $\begin{array}{c}298 \\
K\end{array}$ & $\begin{array}{l}1 \\
M\end{array}$ & $\begin{array}{c}10^{8} \\
\mathrm{~N} / \mathrm{m}\end{array}$ \\
\hline
\end{tabular}

${ }^{a}$ The maximum value of electric potential $\left(|\tilde{\mathbf{E}}|_{\max }\right)$ is far more than the average electric filed in the interelectrode space, due to the closer proximity of the dendritic microstructures to the counterelectrode, as well as the extremely high curvature of the dendrite, reachable to atomic scale; i.e., $|\tilde{\mathbf{E}}|_{\max } \gg \frac{\Delta V}{l}$.

$\cos (\theta)=\sqrt{1-\frac{4 x^{2}}{D^{2}}} ;$ hence,

$$
\delta x^{\prime}=\frac{\delta x}{\sqrt{1-\frac{4 x^{2}}{D^{2}}}}
$$

where $D$ is diameter of the sandwich cell [51].

(4) Starting from the electrode surface, the occupied space by the dendrites has been calculated by the square site percolation paradigm [52].

(5) The infinitesimal calculations have been integrated and normalized to interelectrode distance $\left(\hat{\lambda}_{i}:=\lambda_{i} / l\right)$ to get the dendrite measure $\bar{\lambda}$, as shown in Fig. 5(c), as

$$
\begin{aligned}
\bar{\lambda} & =\frac{1}{\pi D l} \sum_{k=1}^{3} \int_{-\frac{\pi}{3}}^{+\frac{\pi}{3}} \hat{\lambda}_{k}(\theta) \frac{D}{2} d \theta \\
& =\frac{1}{\pi D l} \sum_{k=1}^{3} \int_{-\frac{\pi}{3}}^{+\frac{\pi}{3}} \frac{\hat{\lambda}_{k}(x) d x}{\sqrt{1-\frac{4 x^{2}}{D^{2}}}} .
\end{aligned}
$$

The integral Eq. (34) has been obtained by incremental sum from experimental data. The optimal duty cycle $D$ has been considered where the sensitivity of dendrites metric $\bar{\lambda}$ to duty cycle $D$ is less than $10 \%$. Hence,

$$
\mathbf{D}_{\mathrm{opt}}: \equiv\left\{\frac{\Delta \bar{\lambda}}{\Delta \mathbf{D}} \leqslant 0.1\right\}
$$

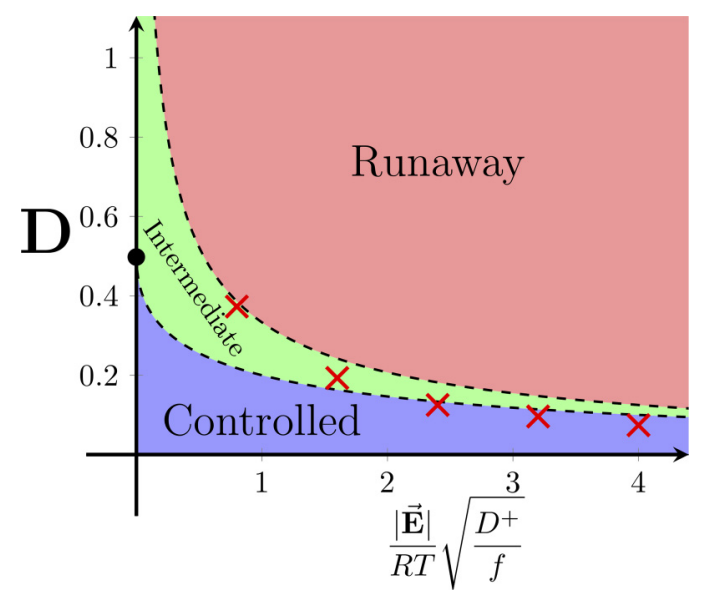

FIG. 6. The regimes based on duty cycle $D$ and frequency $f$ showing the safe/unsafe charging zones. $\times$, Experimental data; $\bullet$, theoretical limit.
Figure 5(b) shows such investigation for the sample pulse frequency of $f=100 \mathrm{~Hz} .^{6}$ The experimental parameters for further data are given in the Table II. ${ }^{7}$

\section{RESULTS AND DISCUSSION}

\section{A. Duty cycle}

Figure 6 visualizes the range of acceptable duty cycle $D$ for the suppression of microstructures. In fact, its theoretical limit can be obtained when the pulse frequency $f$ is increased indefinitely as ${ }^{8}$

$$
\mathbf{D}_{\max }=\lim _{f \rightarrow \infty} \frac{1}{\left(1+\frac{|\overrightarrow{\mathbf{E}}|}{R T} \sqrt{\frac{D^{+}}{2 f}}\right)^{2}+1}=\frac{1}{2} .
$$

Additionally, in Fig. 6, the Controlled region shows the safe zone for pulse charging where the ionic progress in the idle period is competitive enough with the pulse duration. Vice versa, the Runaway region represents the regime where the average ionic lead during pulse wave takes over the rest period, and therefore the dendritic growth would be exacerbated. Nonetheless, the Intermediate region shows the role of random walk where the certainty is less than the other areas. The experimental observations in this figure also illustrate a very high agreement with the analytical trend.

Additionally, it is obvious that the pulse duty cycle $D$ correlates inversely with the diffusion coefficient $D^{+}$and to a higher extend to the magnitude of the electric field $|\overrightarrow{\mathbf{E}}|$. Both parameters exacerbate the growth kinetics and in tradeoff, the duty cycle would have to become more conservative. In fact, the augmentation of electric field in the dendritic tips during the real-time growth causes the quickening growth behavior, which has been addressed before $[31,43]$.

\section{B. Concentration profile}

Looking closer at the depletion-accrual cycle of concentration during full pulse-rest period shown in Fig. 4, we have the

\footnotetext{
${ }^{6}$ While the resolution of some images would not be quite high due to observation conditions from postexperiment acrylic separator, they suffice for binarization purpose shown in Fig. 5(c) and extracting the figure of merit $\bar{\lambda}$.

${ }^{7}$ Note that the current density $i$ and the ionic flux $j$ are correlated with $i=z F j$, where $z$ is the valence number of charge carriers and $F=96.5 \mathrm{kC} / \mathrm{mol}$ is Faraday's constant, representing the amount of charge per mole.

${ }^{8}$ Note that the lower bound has been considered for the inequality to be true in all instances.
} 
TABLE III. The role of curvature on concentration dynamics versus flat surface.

\begin{tabular}{lccccc}
\hline \hline & Curvature & Convex (dendrites) & Concave (pores) \\
\hline Period & $\frac{\partial^{2} C}{\partial r^{2}}$ & $\frac{1}{r} \frac{\partial C}{\partial r}$ & $\frac{\partial C}{\partial t}$ & $\frac{1}{r} \frac{\partial C}{\partial r}$ & $\frac{\partial C}{\partial t}$ \\
Pulse (formation) & - & + & Slower & - & Faster \\
Rest (relaxation) & + & + & Faster & - & Slower \\
\hline \hline
\end{tabular}

following inequality:

$$
\int_{0}^{1} \hat{C}(\hat{r}, t) d \hat{r} \leqslant 1 .
$$

The comparison of the dynamics of ionic concentration, versus the dendrite growth rate indicates that the electrodeposition occurs in a significantly faster kinetics than dendrites growth:

$$
\frac{\partial \hat{C}}{\partial t} \gg \frac{\partial \hat{\lambda}}{\partial t} .
$$

Since the dendrites are the boundary condition for the concentration development per se, such a distinction implies that the concentration profile would occur in the quasi-steadystate regime in the double layer region. This is particularly true during stage of instigation of microstructures, where the nucleation rate is negligible. Therefore, the concentration profile would be obtained by solving the right-hand side of Eq. (22) as

$$
\frac{\partial^{2} \hat{C}}{\partial \hat{r}^{2}}+\frac{\kappa}{r_{d}+\kappa \hat{r}} \frac{\partial \hat{C}}{\partial \hat{r}} \approx 0 .
$$

Setting the boundary condition from Eq. (24) as $\frac{\partial \hat{C}}{\partial \hat{r}}(0, t)=-\frac{\kappa j}{C_{\infty} D^{+}}$, one gets

$$
\frac{d \hat{C}}{d \hat{r}} \approx \frac{-\kappa r_{d} j}{C_{\infty} D^{+}\left(r_{d}+\kappa \hat{r}\right)} .
$$

Integrating again and having $\hat{C}(1, t)=1$ leads to

$$
\hat{C}(\hat{r}, t) \approx 1-\frac{r_{d} j}{C_{\infty} D^{+}} \ln \left(\frac{r_{d}+\kappa}{r_{d}+\kappa \hat{r}}\right) .
$$

For linearization, Eq. (37) can be rearranged as

$$
\hat{C}(\hat{r}, t) \approx 1+\frac{r_{d} j}{C_{\infty} D^{+}} \ln \left(1-\frac{\kappa-\kappa \hat{r}}{r_{d}+\kappa}\right) .
$$

For the mesoscale dendrite the thickness of the double layer $\kappa$ is negligible relative to the radius of the dendrite $r_{d}$ (i.e., $\kappa \ll r_{d}$ ). Therefore, $\frac{\kappa-\kappa \hat{r}}{r_{d}+\kappa} \rightarrow 0$ and $\log$ term can be approximated with the first term of Taylor expansion as 9

$$
\begin{aligned}
\hat{C}(\hat{r}, t) & \approx 1-\frac{r_{d} j}{C_{\infty} D^{+}} \frac{\kappa(1-\hat{r})}{r_{d}+\kappa} \\
& \approx 1-\frac{\kappa j}{C_{\infty} D^{+}}(1-\hat{r}) .
\end{aligned}
$$

Such a linear concentration profile has been addressed in the past for the flat electrodes as well [31]. This profile has been illustrated in Fig. 4 as well. It is obvious that at the reaction

\footnotetext{
${ }^{9}$ By Taylor expansion $\ln (1+\epsilon) \approx \epsilon^{-O}\left(\epsilon^{2}\right),{ }^{0}$, where $0<\epsilon \ll 1$.
}

Sites $(\hat{r} \rightarrow 0)$ the concentration correlates inversely with the ionic flux $j$. To have complete depletion in the reduction sites, we should have the following:

$$
j_{*}=D^{+} \frac{C_{\infty}}{\kappa},
$$

which resembles the flux from the linear concentration distribution throughout the entire span of double layer and has been expressed as the critical current density, where the electrode concentration goes to zero $[53,54]$.

\section{Relaxation time}

Equation (33) in fact resembles the Van Neumann stability criterion for the typical diffusion equation as [55]

$$
\frac{D^{+} \delta t}{\delta r^{2}} \leqslant \frac{1}{2}
$$

The implication shown in Eq. (32) suggests that the relaxation time correlates with the geometric mean of the thickness of the double layer $\kappa$ and the outer radius $r_{O}=\kappa+r_{d}$. The relaxation profile during this time has also been shown in Fig. 4, where the marginal deviation from the absolutely uniform concentration distribution (i.e., where $t_{\mathrm{OFF}} \rightarrow \infty$ ) could be due to the round off error as well as truncation error during the discrete computation [56]. The geometric mean correlation for the relaxation time has been addressed before as the RC time of the system for blocking flat electrodes [42], which implies that the regime of relaxation time would vary across the morphology of the electrodeposits with varying radius of curvature from atomic scale in the dendritic tips, to the completely flat surface in smooth areas $\left(r_{d} \in\left[r_{\text {atom }}, \infty\right]\right)$. Therefore, the homogenized relaxation time would have the following $\operatorname{span}^{10}$ :

$$
\frac{\kappa\left(\kappa+r_{d}\right)}{D^{+}} \leqslant t_{\mathrm{OFF}}^{\mathrm{opt}} \leqslant \frac{\kappa l}{D^{+}} .
$$

Nevertheless, the overall relaxation time of the heterogeneous morphology is determined by the longest relaxation time as the most conservative case, belonging to the flat zones.

\section{Geometry}

As shown in Fig. 3, the convex boundary of dendritic interface is exposed to expanded space in the double-layer medium $\left(r_{O}>r_{d}\right)$. Such geometry alters the dynamics of concentration gradient relative to flat surface during the pulserest cycle. During the pulse period (i.e., formation of concentration gradient) the dendritic sites have limited space

\footnotetext{
${ }^{10}$ The counterelectrode does not geometrywise interfere with the double layer; i.e., $\kappa \ll l$.
} 


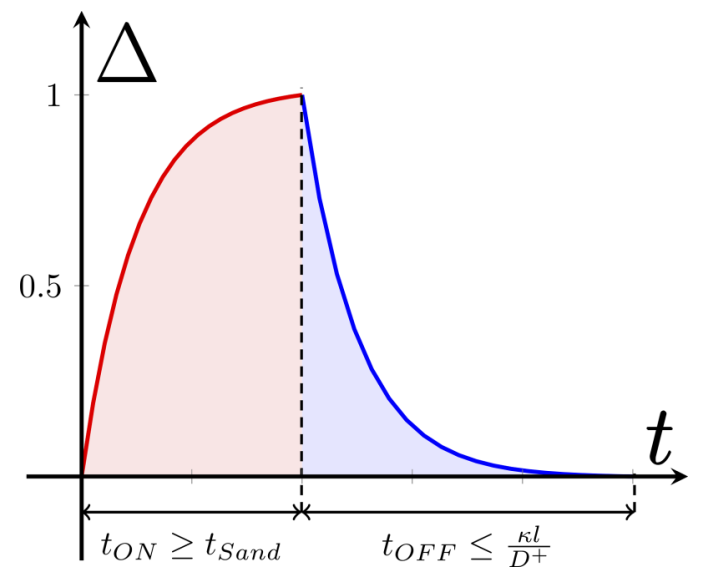

FIG. 7. The time regime for concentration gradient in the convex dendrites.

for the higher feed of ions from the largest space. Hence, the depletion of concentration occurs in slower rate, whereas during the relaxation, there will be larger free domain to diffuse into, relative to flat surface. Therefore, the relaxation occurs with faster rate for convex surfaces. This is also obvious from Eq. (22), where the term $\frac{1}{r} \frac{\partial}{\partial r}$ would alter the concentration dynamics as illustrated in Table III. The sign of the second derivative $\frac{\partial^{2} C}{\partial r^{2}}$ is easily discernible from curvature of the concentration profile in Fig. 4, which is identical for all morphologies concerned [57,58]. For convex dendrites, the curvature term slows down the formation of concentration gradient, whereas it accelerates the relaxation rate. Following the same phenomenology, the relaxation in concave surfaces (i.e., pores) occurs at faster dynamics, as the concentrated atoms have relatively less space to diffuse into. Respectively, the curvature would resist the relaxation for the pores due to lack of space. Such dynamics translates into the number of iterations for convergence in our computations.

The concentration gradient $\vec{\nabla} C$ plays the major role for nonuniform localization of dendritic structures and has a nonlinear behavior in time. During pulse period, as $\vec{\nabla} C$ decreases, the rate of relaxation decreases as well and vanishes when converging to equilibrium (i.e., uniform profile). We define the depletion measure $\Delta(t)$ for tracking its dynamics as

$$
\Delta(t):=1-\hat{C}_{i}(t)
$$

where $\hat{C}_{i}(t)$ is the concentration of the interface (i.e., surface of the dendrite). The variation of depletion measure $\Delta(t)$ during the full pulse-rest period is shown in Fig. 7, assuming that the depletion current density meets the critical value (i.e., $j \geqslant j_{*}$ ) [53]. As is discussed in Table III, the formation of such gradient occurs in a longer time period than the Sand's time [59], whereas the relaxation occurs faster rate (i.e., shorter time) than the flat electrode. This is also obvious from Eq. (40). ${ }^{11}$

\footnotetext{
${ }^{11}$ Obviously based on the geometry of the electrochemical cell. The interelectrode distance is the largest dimension among all the parameters considered. Therefore, $r_{d}+\kappa \leqslant l$.
}

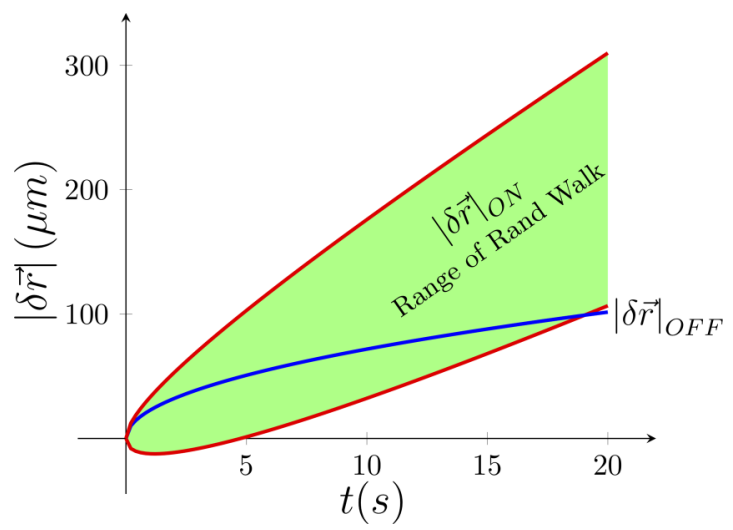

FIG. 8. Extended range of random walk during pulse interval, compared with the progress of diffusion wave in the rest period.

Looking at the extended range of diffusion-migration dynamics at provided more insight to the range of duty cycle. The diffusion length scales with square root of time $\left(\delta \vec{r}_{D} \propto t^{\frac{1}{2}}\right)$, whereas the migration lead scales linearly with it $\left(\delta \vec{r}_{M} \propto t\right)$. Therefore, one expects that given a sufficient time $\delta t_{\text {eq }}$, the migration front would take over the diffusion lead. The hypothetical comparison of the progress of sole-diffusive and sole-migrative waves is possible from Eqs. (1) and (2) combined with the Einstein relationship $\left(D^{+}=\mu^{+} R T\right)$ [34]:

$$
\delta t_{\mathrm{eq}}=\frac{2 R T}{\mu^{+}|\overrightarrow{\mathbf{E}}|^{2}},
$$

where $R$ is gas constant ${ }^{12}$ and $T$ is temperature. Closer look at the dynamics of progress during pulse and rest periods from Eq. (9) leads to Fig. 8. It is clear that during the initial moments, the progress in the rest period could be more competitive with the pulse time. From the Eq. (13), initially, the idle ratio $\gamma$ decays exponentially versus the dimension-less charge period $t_{\mathrm{ON}}$. The exponential decay behavior indicates that relatively shorter amount of idle ratio is needed so that the diffusion lead would catch up the progress during applied pulse period. This is also obvious from Eq. (12), as the term $\sqrt{\gamma}$ is comparable and in the order of $\gamma$. As the pulse period $t_{\mathrm{ON}}$ increases, by neglecting the lower order terms, we reach to the limit $\gamma \propto t_{\mathrm{ON}}$, which directly means $t_{\mathrm{OFF}} \propto t_{\mathrm{ON}}^{2}$, therefore for higher applied pulse period $t_{\mathrm{ON}}$, the equivalent idle period $t_{\mathrm{OFF}}$ for concentration relaxation has to be significantly higher. As well in Eq. (15) if $t_{\mathrm{ON}}$ increase indefinitely, the correlation of the needed rest period $t_{\mathrm{OFF}}$ for the given pulse period $t_{\mathrm{ON}}$ will move toward linear relationship from exponential decay behavior. On the other extreme, the application of indefinitely high pulse frequency $f$ (i.e., $t_{\mathrm{ON}} \rightarrow 0$ ) might not let the ions reach the reaction sites. Therefore, the fine-enough pulse period would make the applied rest period for the charge relaxation easier to be competitive with it, as depicted in Fig. 6.

\footnotetext{
${ }^{12} \mathrm{R}=8.314 \mathrm{~J} / \mathrm{mol} \mathrm{K}$
} 


\section{CONCLUSIONS}

In this paper, we have performed analytical developments from stochastic ionic dynamics for the effective suppression of growing dendritic microstructures during electrodeposition. We defined such square pulse charging parameters in terms of the range of pulse duty cycle $D$ and the respective idle time period $t_{\mathrm{OFF}}$. Our model considers the localizations of both ionic concentration and electric field within the interface of the electrochemical cell, where the nonlinear role of the dendrite curvature on the relaxation is demonstrated in terms of cell geometry and the transport property of the electrolyte solution. The results are useful for estimating the effective charging for dendrite-prone electrochemical environments, particularly those of involving metallic electrodes (i.e., lithium, etc.).

\section{ACKNOWLEDGMENTS}

The authors thank the Bill and Melinda Gates Foundation for financial support (Grant No. OPP1192374) and Bahçeşehir University for the BAP support. Additionally, the insightful discussions with Dr. Agustin Colussi (Caltech), Dr. Jaime Marian (UCLA), and Dr. Martin Bazant (MIT) are acknowledged.
[1] Z. Li, J. Huang, B. Y. Liaw, V. Metzler, and J. Zhang, A review of lithium deposition in lithium-ion and lithium metal secondary batteries, J. Power Sources 254, 168 (2014).

[2] P. Pei, K. Wang, and Z. Ma, Technologies for extending zinc air batterys cyclelife: A review, Appl. Energy 128, 315 (2014).

[3] M. D. Slater, D. Kim, E. Lee, and C. S. Johnson, Sodium-ion batteries, Adv. Funct. Mater. 23, 947 (2013).

[4] S. Li, J. Yang, and Y. Lu, Lithium metal anode, Encyclopedia of Inorganic and Bioinorganic Chemistry (Wiley, Hoboken, NJ, 2012), pp. 1-21.

[5] W. Xu, J. L. Wang, F. Ding, X. L. Chen, E. Nasybutin, Y. H. Zhang, and J. G. Zhang, Lithium metal anodes for rechargeable batteries, Energy Environ. Sci. 7, 513 (2014).

[6] K. Xu, Nonaqueous liquid electrolytes for lithium-based rechargeable batteries, Chem. Rev. Columbus 104, 4303 (2004).

[7] Y. Li and H. Dai, Recent advances in zinc-air batteries, Chem. Soc. Rev. 43, 5257 (2014).

[8] F. Orsini, A. D. Pasquier, B. Beaudoin, J. M. Tarascon et al., In situ scanning electron microscopy observation of interfaces with plastic lithium batteries, J. Power Sources 76, 19 (1998).

[9] C. Monroe and J. Newman, The effect of interfacial deformation on electrodeposition kinetics, J. Electrochem. Soc. 151, A880 (2004).

[10] C. P. Nielsen and H. Bruus, Morphological instability during steady electrodeposition at overlimiting currents, Phys. Rev. E 92, 052310 (2015).

[11] P. P. Natsiavas, K. Weinberg, D. Rosato, and M. Ortiz, Effect of prestress on the stability of electrode-electrolyte interfaces during charging in lithium batteries, J. Mech. Phys. Solids 95, 92 (2016).

[12] K. J. Harry, D. T. Hallinan, D. Y. Parkinson, A. A. MacDowell, and N. P. Balsara, Detection of subsurface structures underneath dendrites formed on cycled lithium metal electrodes, Nat. Mater. 13, 69 (2014).

[13] J. Steiger, D. Kramer, and R. Monig, Mechanisms of dendritic growth investigated by in situ light microscopy during electrodeposition and dissolution of lithium, J. Power Sources 261, 112 (2014).

[14] N. Schweikert, A. Hofmann, M. Schulz, M. Scheuermann, S. T. Boles, T. Hanemann, H. Hahn, and S. Indris, Suppressed lithium dendrite growth in lithium batteries using ionic liquid electrolytes: Investigation by electrochemical impedance spectroscopy, scanning electron microscopy, and in situ li-7 nuclear magnetic resonance spectroscopy, J. Power Sources 228, 237 (2013).

[15] R. Younesi, G. M. Veith, P. Johansson, K. Edström, and T. Vegge, Lithium salts for advanced lithium batteries: Li-metal, Li-o 2, and Li-s, Energy Environ. Sci. 8, 1905 (2015).

[16] C. Brissot, M. Rosso, J. N. Chazalviel, and S. Lascaud, In situ concentration cartography in the neighborhood of dendrites growing in lithium/polymer-electrolyte/lithium cells, J. Electrochem. Soc. 146, 4393 (1999).

[17] I. W. Seong, C. H. Hong, B. K. Kim, and W. Y. Yoon, The effects of current density and amount of discharge on dendrite formation in the lithium powder anode electrode, J. Power Sources 178, 769 (2008).

[18] G. M. Stone, S. A. Mullin, A. A. Teran, D. T. Hallinan, A. M. Minor, A. Hexemer, and N. P. Balsara, Resolution of the modulus versus adhesion dilemma in solid polymer electrolytes for rechargeable lithium metal batteries, J. Electrochem. Soc. 159, A222 (2012).

[19] A. Aryanfar, T. Cheng, A. J. Colussi, B. V. Merinov, W. A. Goddard III, and M. R. Hoffmann, Annealing kinetics of electrodeposited lithium dendrites, J. Chem. Phys. 143, 134701 (2015).

[20] Y. Yao, X. Zhao, A. A. Razzaq, Y. Gu, X. Yuan, R. Shah, Y. Lian, J. Lei, Q. Mu, Y. Ma et al., Mosaic rGO layer on lithium metal anodes for effective mediation of lithium plating and stripping, J. Mater. Chem. A 7, 12214 (2019).

[21] J. Qian, Y. Li, M. Zhang, R. Luo, F. Wang, Y. Ye, Y. Xing, W. Li, W. Qu, L. Wang et al., Protecting lithium/sodium metal anode with metal-organic framework based compact and robust shield, Nano Energy 60, 866 (2019).

[22] W. Deng, W. Zhu, X. Zhou, F. Zhao, and Z. Liu, Regulating capillary pressure to achieve ultralow areal mass loading metallic lithium anodes, Energy Storage Mater. (2019), doi:10.1016/j.ensm.2019.02.027.

[23] A. W. Abboud, E. J. Dufek, and B. Liaw, Implications of local current density variations on lithium plating affected by cathode particle size, J. Electrochem. Soc. 166, A667 (2019).

[24] C. Xu, Z. Ahmad, A. Aryanfar, V. Viswanathan, and J. R. Greer, Enhanced strength and temperature dependence of mechanical properties of $\mathrm{Li}$ at small scales and its implications for Li metal anodes, Proc. Natl. Acad. Sci. USA 114, 57 (2017).

[25] P. Wang, W. Qu, W.-L. Song, H. Chen, R. Chen, and D. Fang, Electrochemomechanical issues at the interfaces in solid-state lithium metal batteries, Adv. Funct. Mater. 25, 1900950 (2019). 
[26] R. Bhattacharyya, B. Key, H. L. Chen, A. S. Best, A. F. Hollenkamp, and C. P. Grey, In situ nmr observation of the formation of metallic lithium microstructures in lithium batteries, Nat. Mater. 9, 504 (2010).

[27] S. Chandrashekar, N. M. Trease, H. J. Chang, L.-S. Du, C. P. Grey, and A. Jerschow, 7li mri of Li batteries reveals location of microstructural lithium, Nat. Mater. 11, 311 (2012).

[28] Y. Li and Y. Qi, Energy landscape of the charge transfer reaction at the complex Li/SEI/electrolyte interface, Energy \& Environ. Sci. 12, 1286 (2019).

[29] L. M. Kasmaee, A. Aryanfar, Z. Chikneyan, M. R. Hoffmann, and A. J. Colussi, Lithium batteries: Improving solid-electrolyte interphases via underpotential solvent electropolymerization, Chem. Phys. Lett. 661, 65 (2016).

[30] J. N. Chazalviel, Electrochemical aspects of the generation of ramified metallic electrodeposits, Phys. Rev. A 42, 7355 (1990).

[31] C. Monroe and J. Newman, Dendrite growth in lithium/polymer systems-A propagation model for liquid electrolytes under galvanostatic conditions, J. Electrochem. Soc. 150, A1377 (2003).

[32] T. A. Witten and L. M. Sander, Diffusion-limited aggregation, Phys. Rev. B 27, 5686 (1983).

[33] R. Zhang, X. Shen, X.-B. Cheng, and Q. Zhang, The dendrite growth in $3 \mathrm{D}$ structured lithium metal anodes: Electron or ion transfer limitation? Energy Storage Mater. (2019), doi:10.1016/j.ensm.2019.03.029.

[34] A. J. Bard and L. R. Faulkner, Electrochemical Methods: Fundamentals and Applications (Wiley, Hoboken, NJ, 1980).

[35] D. Tewari and P. P. Mukherjee, Mechanistic understanding of electrochemical plating and stripping of metal electrodes, J. Mater. Chem. A 7, 4668 (2019).

[36] M. Z. Bazant, B. D. Storey, and A. A. Kornyshev, Double Layer in Ionic Liquids: Overscreening versus Crowding, Phys. Rev. Lett. 106, 046102 (2011).

[37] V. Fleury, Branched fractal patterns in nonequilibrium electrochemical deposition from oscillatory nucleation and growth, Nature 390, 145 (1997).

[38] A. Aryanfar, D. J. Brooks, A. J. Colussi, B. V. Merinov, W. A. Goddard III, and M. R. Hoffmann, Thermal relaxation of lithium dendrites, Phys. Chem. Chem. Phys. 17, 8000 (2015).

[39] J. Li, E. Murphy, J. Winnick, and P. A. Kohl, The effects of pulse charging on cycling characteristics of commercial lithium-ion batteries, J. Power Sources 102, 302 (2001).

[40] M. S. Chandrasekar and M. Pushpavanam, Pulse and pulse reverse plating-Conceptual advantages and applications, Electrochim. Acta 53, 3313 (2008).

[41] A. Aryanfar, D. Brooks, B. V. Merinov, W. A. Goddard Iii, A. J. Colussi, and M. R. Hoffmann, Dynamics of lithium dendrite growth and inhibition: Pulse charging experiments and Monte Carlo calculations, J. Phys. Chem. Lett. 5, 1721 (2014).
[42] M. Z. Bazant, K. Thornton, and A. Ajdari, Diffuse-charge dynamics in electrochemical systems, Phys. Rev. E 70, 021506 (2004).

[43] A. Aryanfar, D. J. Brooks, and W. A. Goddard, Theoretical pulse charge for the optimal inhibition of growing dendrites, MRS Adv. 3, 1201 (2018).

[44] S. Chandrashekar, O. Oparaji, G. Yang, and D. Hallinan, Communication 7li mri unveils concentration dependent diffusion in polymer electrolyte batteries, J. Electrochem. Soc. 163, A2988 (2016).

[45] J. Philibert, One and a half century of diffusion: Fick, Einstein, before and beyond, Diffus. Fund. 4, 1 (2006).

[46] P. J. Pritchard, J. W. Mitchell, and J. C. Leylegian, Fox and McDonald's Introduction to Fluid Mechanics, Binder Ready Version (John Wiley \& Sons, Hoboken, NJ, 2016).

[47] M. A. Khamsi and W. A. Kirk, An Introduction to Metric Spaces and Fixed Point Theory (John Wiley \& Sons, Hoboken, NJ, 2011), Vol. 53.

[48] J. L. Barton and J. O'M Bockris, The electrolytic growth of dendrites from ionic solutions, Proc. R. Soc. London A 268, 485 (1962).

[49] A. Aryanfar, Method and device for dendrite research and discovery in batteries, US Patent No. 9, 620, 808 (2017).

[50] N. Otsu, A threshold selection method from gray-level histograms, Automatica 11, 23 (1975).

[51] A. Aryanfar, D. J. Brooks, A. J. Colussi, and M. R. Hoffmann, Quantifying the dependence of dead lithium losses on the cycling period in lithium metal batteries, Phys. Chem. Chem. Phys. 16, 24965 (2014).

[52] A. Aryanfar, W. Goddard III, and J. Marian, Constriction percolation model for coupled diffusion-reaction corrosion of zirconium in PWR, Corros. Sci. 158, 108058 (2019).

[53] C. Brissot, M. Rosso, J. N. Chazalviel, and S. Lascaud, Dendritic growth mechanisms in lithium/polymer cells, J. Power Sources 81, 925 (1999).

[54] Y. Chen, Y. Luo, H. Zhang, C. Qu, H. Zhang, and X. Li, The challenge of lithium metal anodes for practical applications, Small Methods 3, 1800551 (2019).

[55] E. Isaacson and H. B. Keller, Analysis of Numerical Methods (Courier Corporation, North Chelmsford, MA, 2012).

[56] W. Edmund Milne and W. E. Milne, Numerical Solution of Differential Equations (Wiley, New York, 1953), Vol. 19.

[57] C. Leger, J. Elezgaray, and F. Argoul, Dynamical characterization of one-dimensional stationary growth regimes in diffusionlimited electrodeposition processes, Phys. Rev. E 58, 7700 (1998).

[58] C. Leger, J. Elezgaray, and F. Argoul, Experimental Demonstration of Diffusion-Limited Dynamics in Electrodeposition, Phys. Rev. Lett. 78, 5010 (1997).

[59] M. Rosso, Electrodeposition from a binary electrolyte: New developments and applications, Electrochim. Acta 53, 250 (2007). 